\title{
Evaluation of a novel reference chamber "stealth chamber" through Monte Carlo simulations and experimental data
}

\author{
Luis Alberto Vazquez Quino, Claudia Ivette Huerta Hernandez, Oscar Calvo, Mark Deweese
}

Alyzen Medical Physics, Inc., 1801 S 54th Street, Paragould, Arkansas, USA

Received March 11, 2015; Revised April 20, 2015; Accepted May 03, 2015; Published Online June 01, 2015

\section{Original Article}

\begin{abstract}
Purpose: To evaluate a novel reference chamber (Stealth Chamber by IBA) through experimental data and Monte Carlo simulations for 6 and 15 MV photon energies. Methods: Monte Carlo simulations in a water phantom for field sizes ranging from $3 \times 3$ and $25 \times 25 \mathrm{~cm}^{2}$ were performed for both energies with and without the Monte Carlo model of the Stealth Chamber in the beam path, and compared to commissioning beam data. Percent depth doses (PDDs), profiles, and gamma analysis of the simulations were performed along with an energy spectrum analysis of the phase-space files generated during the simulation. Experimental data were acquired in water with IBA three-dimensional (3D) blue phantom in a set-up identical to the one used in the Monte Carlo simulations. PDD comparisons for fields ranging from $1 \times 1$ to $25 \times 25 \mathrm{~cm}^{2}$ were performed for photon energies. Profile comparison for fields ranging from $1 \times 1$ to $25 \times 25 \mathrm{~cm}^{2}$ were executed for the depths of dmax, 5, 10 and $20 \mathrm{~cm}$. Criteria of $1 \%, 1 \mathrm{~mm}$ to compare PDDs and profiles were used. Transmission measurements with the Stealth Chamber and a Matrixx detector from IBA were investigated. Measurements for 6 and $15 \mathrm{MV}$ with fields ranging from $3 \times 3$ to $25 \times 25 \mathrm{~cm}^{2}$ dimensions were acquired in an open field with and without the Stealth Chamber in the path of the beam. Profiles and gamma analysis with a $1 \%, 1 \mathrm{~mm}$ gamma analysis criterion were performed. Results: Monte Carlo simulations of the PDDs and profiles demonstrate the agreement between both simulations. Furthermore, the gamma analysis $(1 \%, 1 \mathrm{~mm})$ result of the comparison of both planes has $100 \%$ of the points passing the criteria. The spectral distribution analysis of the phase spaces for an open field with and without the chamber reveals the agreement between both simulations. Experimental measurements of PDDs and profiles have been conducted and reveal the comparability of relative dosimetric data acquired with the Stealth Chamber and our gold standard the CC13 chamber. Transmission data measured with an ion chamber array (Matrixx) showed the small attenuation caused by the use of the Stealth Chamber. Conclusion: Simulations and experimental results from this investigation indicate the benefits associated with chamber positioning and time expended during the acquisition of the relative measurements of PDDs and profiles for the beam commissioning of photon beams when the Stealth Chamber is used as a reference chamber to perform these tasks. The results demonstrate that relative profiles and PDDs scanned with the Stealth Chamber in place are consistent with those made using a CC13 chamber within a $1 \%$ and $1 \mathrm{~mm}$ criterion.
\end{abstract}

Keywords: Reference Chamber; Monte Carlo Simulations; Linac Commissioning; Ion Chamber

\section{Introduction}

During the commissioning of a linear accelerator (linac), physicists face the challenge of acquiring accurate beam data. ${ }^{1}$ Among all of the beam data required to be collected, the measurement of percent depth doses (PDDs) and profiles for a small field size $\left(<3 \times 3 \mathrm{~cm}^{2}\right)$ is one of the most challenging tasks due mainly to the position and use of a reference chamber. The nature of small-field measurements, and, the dimensions and position of the reference chamber in the beam path, together with the selection of an appropriate detector in order to avoid partial-volume effects, represent a major challenge for physicists. At the time, little information can be found in the literature regarding the characteristics and advantages of the use of a reference chamber in data acquisition for the relative dose measurements of PDDs and profiles in water. ${ }^{2}$

In order to assess this issue, a reference chamber virtually transparent to the beam has been designed by IBA (IBA Dosimetry GmbH Schwarzenbruck, Germany) and is called a "Stealth Chamber," as shown in Figure 1. The Stealth Chamber has a thickness equivalent of $0.5 \mathrm{~mm}$ of aluminum and a rectangular design that can be used in the data acquisition of fields ranging from $0.5 \times 0.5 \mathrm{~cm}^{2}$ to a maximum field size of $25 \times 25 \mathrm{~cm}^{2}$. The equivalent thickness of the chamber studied makes it very attractive to be used as reference chamber due to the small attenuation caused to the beam and

Corresponding author: Luis Alberto Vazquez-Quino; Alyzen Medical Physics, Inc., 1801 S 54th Street, Paragould, Arkansas, USA.

Cite this article as: Vazquez-Quino L, Huerta-Hernandez C, Calvo 0 , Deweese M. Evaluation of a novel reference chamber "stealth chamber" through Monte Carlo simulations and experimental data. Int J Cancer Ther Oncol 2015; 3(2):3222. D0I: 10.14319/ijcto.32.22 
the spatially uniform attenuation. Because of the Stealth Chamber's ability to be attached to the head of the linac, no repositioning is needed. This feature eliminates the need for a physicist to enter the room, thus saving valuable time during data collection.

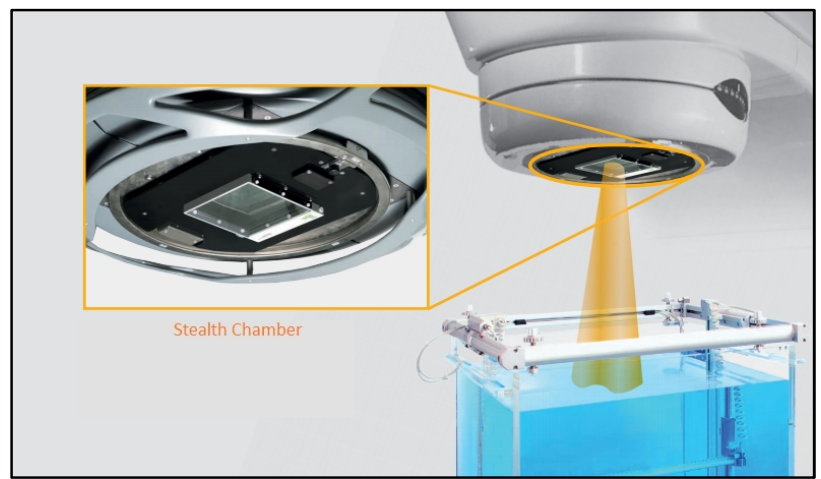

FIG. 1: Stealth chamber set up on head of linac (Courtesy of IBA Dosimetry GmbH Schwarzenbruck, Germany).

In the present work, we aimed to characterize and evaluate the Stealth Chamber through a theoretical characterization via Monte Carlo simulations and experimental characterization with PDDs and profiles as well as ionometric and fluence measurements. AAPM TG-105 recommends that Monte Carlo simulations be performed under the same conditions as the measurements. ${ }^{3-4}$ In addition, Monte Carlo simulations provide the most accurate dose predictions for small-field dosimetry, as reported in literature, especially for fields of the order of less than $3 \times 3 \mathrm{~cm}^{2} .^{5-28}$

Through the investigation conducted, the authors aim to increase the literature available about the use of a reference chamber during data collection. At this moment, just two studies on the Stealth Chamber have been reported in the literature, one by Vazquez et al., and the other by Gersh. ${ }^{10,29}$

\section{Methods and Materials}

\section{Monte Carlo simulations}

Monte Carlo simulations for this investigation were performed in two steps. First, a phase space of the linac head was obtained with and without the Monte Carlo model of the Stealth Chamber in the geometry built according to manufacturer specifications. Secondly, the phase spaces obtained from these simulations were used in the dose calculation simulations in the water phantom.

\section{BEAMnrc simulations}

Using the phase-space files for TrueBeam photon beams available by Varian (Varian Medical Systems, Palo Alto, CA) in International Atomic Energy Agency (IAEA)-compliant format for 6 and $15 \mathrm{MV}$, Monte Carlo simulations were performed using BEAMnrc simulations and DOSXYZnrc (NRC, Canada) dose simulations in order to investigate the pertur- bation introduced by the Stealth Chamber on PDDs and profiles measured in a water tank. Field sizes for $3 \times 3$ and $5 \times 5$ $\mathrm{cm}^{2}$ were simulated for both energies with and without the Stealth Chamber, which was built according to specifications, in a water phantom of $30 \times 30 \times 30 \mathrm{~cm}^{3}$ and a pixel resolution of $2 \mathrm{~mm}$. The PDDs, profiles, and gamma analysis of the simulations were performed along with an energy spectrum analysis of the phase-space files generated during the simulation.

Simulations of the linac head were structured in the following order: phase space of the simulations from Varian until the region superior to the jaw region for 6 and $15 \mathrm{MV}$, jaws (tungsten), Stealth Chamber (for chamber perturbation investigation). The materials used in the simulations were extracted from the 700 ICRU PEGS4 (Preprocessor for Electron Gamma Shower), which are available in BEAMnrc. For electrons a cutoff energy of ECUT $=700 \mathrm{keV}$ and for photons PCUT $=10 \mathrm{keV}$ were used (8).

\section{DOSXYZnrc simulations}

Dose calculations in DOSXYZnrc Monte Carlo package were performed with the phase space files described above as input sources. As in BEAMnrc simulations the ECUT energy used was $700 \mathrm{KeV}$, and the PCUT energy was $10 \mathrm{KeV}$. Figure 2 shows the geometry used in the dose calculation for the simulations. A water phantom of $30 \times 30 \times 30 \mathrm{~cm}^{3}$ was built in DOSXYZnrc with a voxel dimension of $2 \times 2 \times 2 \mathrm{~mm}^{3}$. The number of histories simulated in DOSXYZnrc was established in order to achieve a statistical uncertainty in the 20 highest doses calculated of less than $1 \%$.

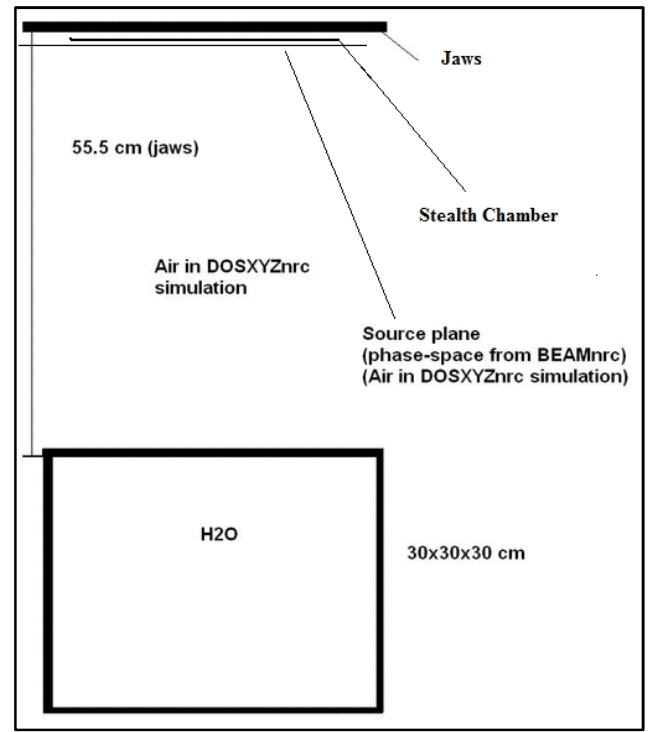

FIG. 2: Water phantom dose calculation in DOSXYZnrc.

\section{Evaluation of Monte Carlo simulations}

3D dose files of the water simulations were obtained as the output of the DOSXYZnrc. An in-house MATLAB programming language routine (The MathWorks, Inc., Natick, Massachusetts, USA) was developed to convert 3D dose files 
into ASCII files to be imported into Omnipro software from IBA. Dose planes of the simulations were imported into Omnipro to perform analysis of profiles and gamma analysis of the simulations. A local dose criterion of $1 \%$ and $1 \mathrm{~mm}$ was set for gamma analysis. Furthermore, a spectral distribution (energy spectrum) analysis was conducted in the Beamdp software from the BEAMnrc package. The analysis was performed in the phase spaces from the linac head in which an open field energy spectrum was compared to the energy spectrum of an open field with the Stealth Chamber in its path.

\section{Experimental data acquisition}

Measurements in water with IBA 3D blue phantom were performed. A setup similar to the one used in the Monte Carlo simulations was used in the protocol of data acquisition, and a source-to-surface distance (SSD) of $100 \mathrm{~cm}$ was chosen for all measurements. All measurements were performed with a Varian TrueBeam linac for 6 and 15 MV photon energies. For an ion chamber, a CC13 from IBA was used as our gold standard for water measurements. The CC13ion chamber has an active volume of $0.13 \mathrm{cc}$ and an inner radius of $3 \mathrm{~mm}$. In this work, CC13 measurements refer to CC13 chambers used as the field and reference chamber in the measurements. For measurements with the Stealth Chamber as the reference chamber, a CC13 chamber was set as the field chamber (see Figure 1). This gave us the freedom to compare measurements with the same chamber for field measurements with the same dimensions and the exact same conditions.

PDDs and profiles at the depths of dmax, 5, 10, and $20 \mathrm{~cm}$ were measured during data acquisition for 6 and $15 \mathrm{MV}$ photon beams. PDD comparison for fields ranging from $1 \times 1$ to $25 \times 25 \mathrm{~cm}^{2}$ were performed. Profile comparison for fields ranging from $1 \times 1$ to $25 \times 25 \mathrm{~cm}^{2}$ were executed for the depths of dmax, 5, 10, and $20 \mathrm{~cm}$. A Criterion of $1 \%, 1 \mathrm{~mm}$ was used to compare PDDs and profiles.

Furthermore, transmission measurements were performed with the Stealth Chamber and a Matrixx detector from IBA, which has an active area of 24.4 by $24.4 \mathrm{~cm}^{2}$, a 1020 chamber arrangement, and a resolution of $7.62 \mathrm{~mm}$. Measurements for 6 and $15 \mathrm{MV}$ with a $10 \times 10 \mathrm{~cm}^{2}$ field were acquired with an open field. A second set of measurements with same open-field conditions as above was performed but this time with the Stealth Chamber in the path of the beam.

Analysis of the transmission measurements for the Stealth Chamber was performed in the Omnipro software from IBA. Profiles and gamma analysis with a passing criterion of $1 \%$ and $1 \mathrm{~mm}$ used for our transmission data comparison. The first goal of the transmission data was to quantify the amount of attenuation that the Stealth Chamber provided while on the path of the beam. Secondly, the transmission data were used to demonstrate that for relative comparison, the use of the Stealth Chamber would not introduce a large perturbation to the beam. After the data were normalized, the passing criterion for all of the points in our measurements was a gamma of a $1 \%$ dose difference and a $1 \mathrm{~mm}$ distance to agreement (DTA).

\section{Results}

\section{Monte Carlo simulations}

The Monte Carlo model was evaluated through comparison to commissioning data of the TrueBeam machine used in the investigation. PDDs and profiles at depth of dmax, 5, 10, and $20 \mathrm{~cm}$, acquired during linac commissioning were compared against our Monte Carlo data in order to benchmark our model. PDDs and profiles were within a $1 \%$ dose difference and a $1 \mathrm{~mm}$ DTA between our Monte Carlo model and the beam commissioning data. An example of the data agreement is presented in Figure 3 for a PDD comparison for a $6 \mathrm{MV}$ with $3 \times 3$ and $5 \times 5 \mathrm{~cm}^{2}$ data comparison.

Figure 4 shows the results for a $6 \mathrm{MV}$ beam and a $3 \times 3 \mathrm{~cm}^{2}$ field with and without the reference chamber in its beam path. Results of the PDDs and profiles demonstrate agreement between both simulations and the commissioning beam data. Furthermore the gamma analysis $(1 \%, 1 \mathrm{~mm})$ results of the comparison of both planes have $100 \%$ of the points passing the criteria. Similar results were obtained from the rest of the simulations with a 6 and $15 \mathrm{MV}$ photon beam, with $3 \times 3$ and $5 \times 5 \mathrm{~cm}^{2}$ fields.

The results of the analysis of the spectral distribution to assess the perturbation introduced by the Stealth Chamber as a reference chamber are summarized in Figure 5. The spectral distribution analysis of the phase spaces for an open field with and without the chamber reveals the agreement between both simulations. The close match between these simulations for the energy spectrum analysis illustrates the very small perturbation introduced into the beam when the Stealth Chamber is used as a reference chamber.

\section{Stealth chamber and physical measurements in water} PDD curves obtained from measurements with the Stealth Chamber and the CC13 as the reference chamber were compared to each other for field sizes ranging from $1 \times 1$ to $25 \times 25$ $\mathrm{cm}^{2}$ as described above for 6 and $15 \mathrm{MV}$. The PDD comparison is shown in Figures 5 and $\mathbf{6}$ below. Both curves agree within a criterion of $1 \%, 1 \mathrm{~mm}$ of each other. Normalized PDD comparison is presented in Figures 6 and 7, and no difference can be observed for relative measurements acquired with the CC13 chamber or the Stealth Chamber as a reference chamber in the measurements. Similar results have been observed in the rest of the field measured in this investigation with a criterion of $1 \%, 1 \mathrm{~mm}$ DTA. 


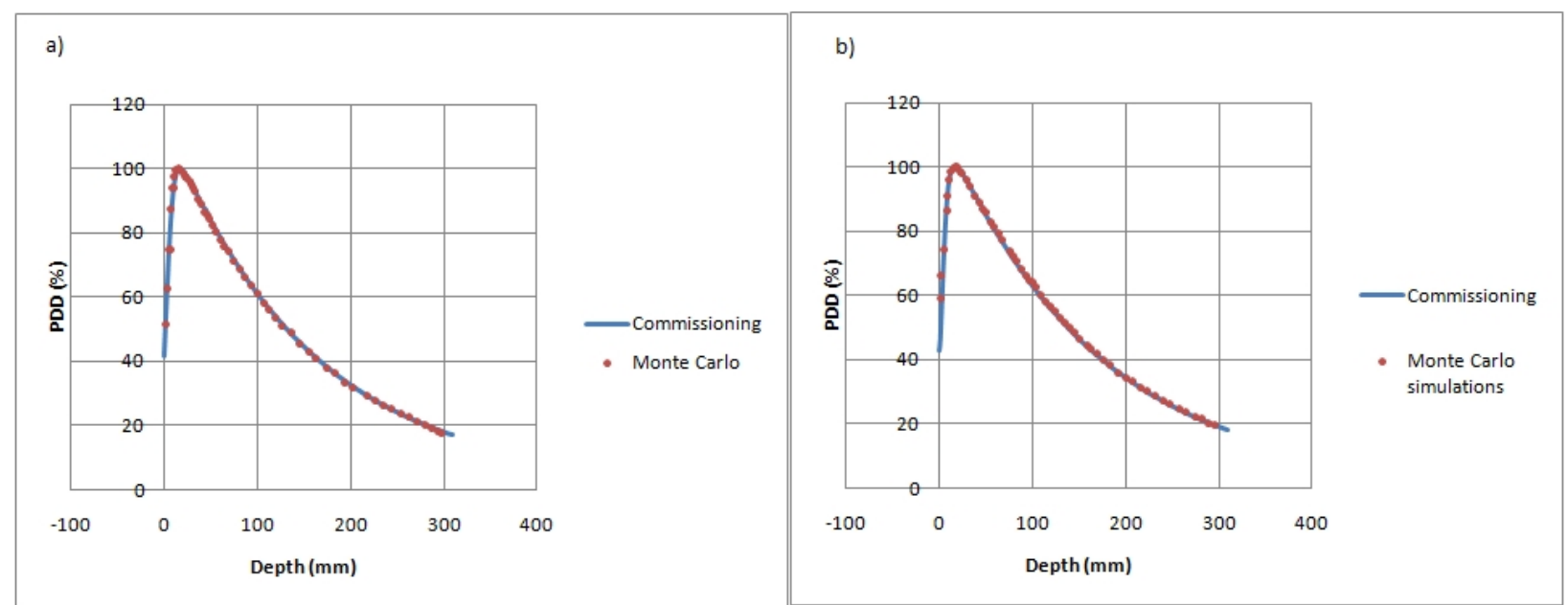

FIG. 3: Monte Carlo model PDD comparison against commissioning data for a) $6 \mathrm{MV}, 3 \times 3 \mathrm{~cm}^{2}$ field and b) $6 \mathrm{MV}, 5 \times 5 \mathrm{~cm}^{2}$ field.

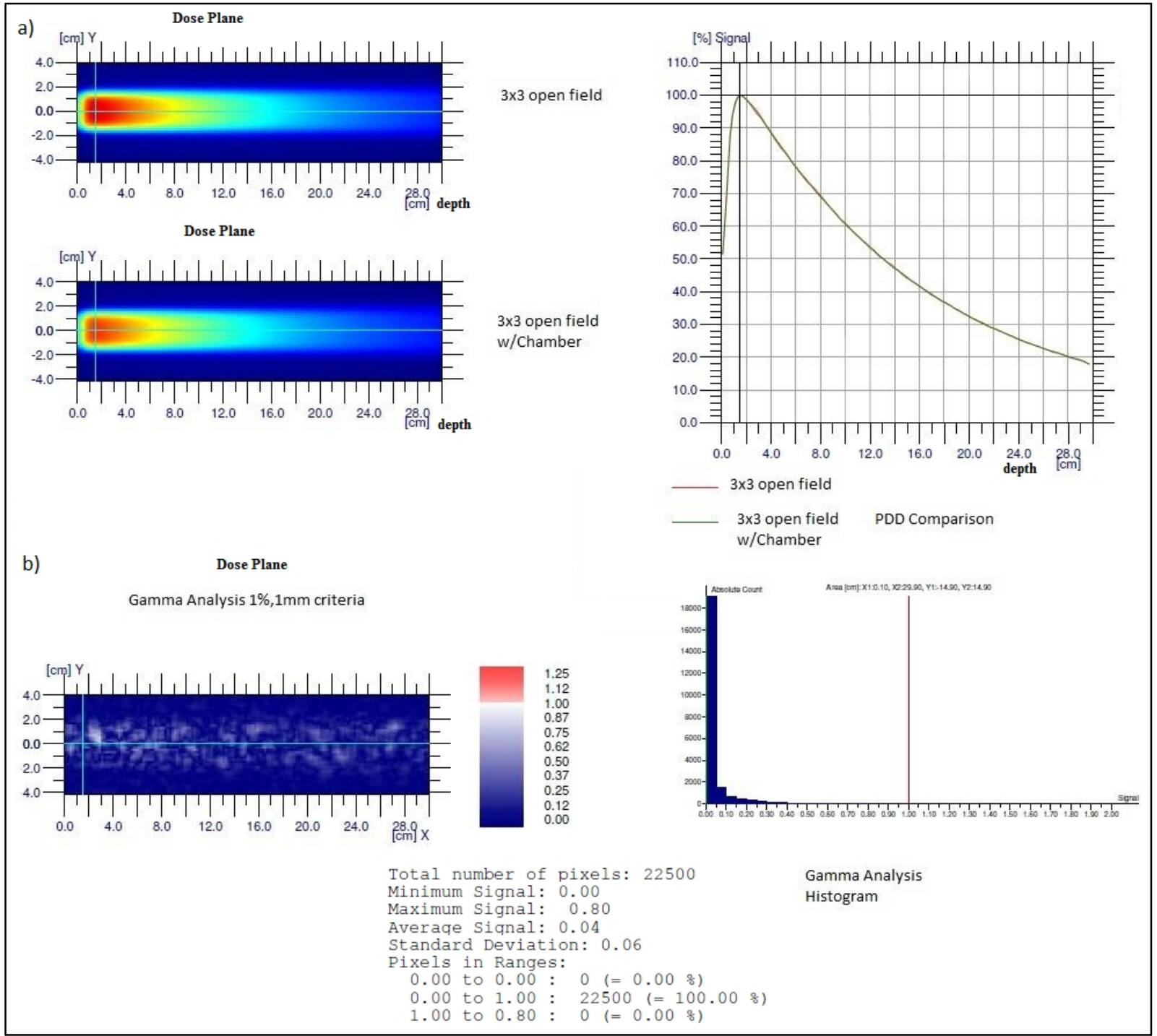

FIG. 4: a) Profile and b) gamma analysis of Montecarlo Simulation data for a field size of $3 \times 3 \mathrm{~cm}^{2}$ at $6 \mathrm{MV}$ with and without the Stealth chamber. 


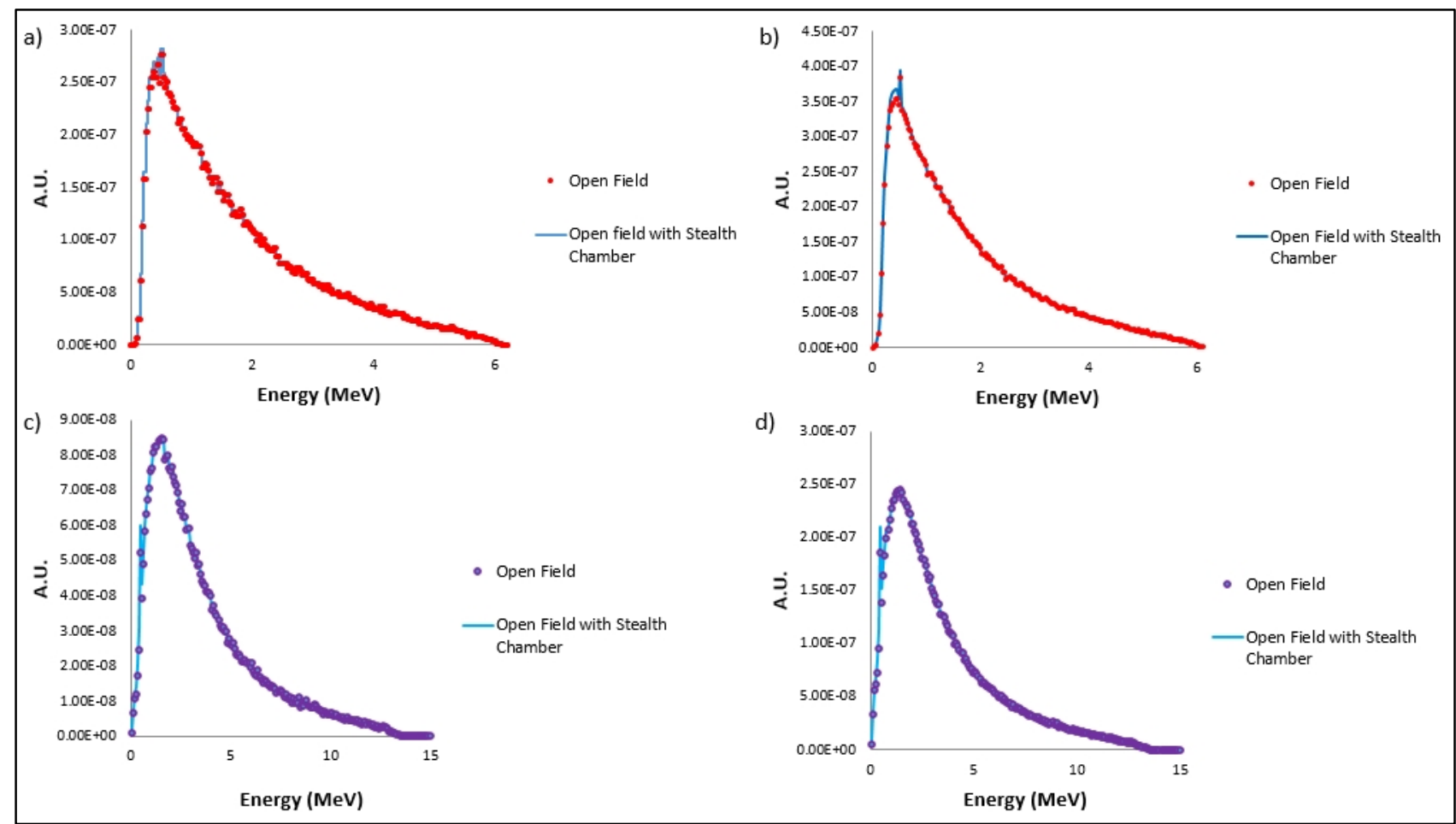

FIG. 5: Spectral distribution (Energy spectrum) analysis of the Phase spaces at $6 \mathrm{MV}$ a) $3 \times 3 \mathrm{~cm}^{2}$ b) $5 \times 5 \mathrm{~cm}^{2}$ and $15 \mathrm{MV}$ c) $3 \times 3 \mathrm{~cm}^{2}$ d) $5 \times 5 \mathrm{~cm}^{2}$ for an open field with and without the stealth chamber on the path of the beam.

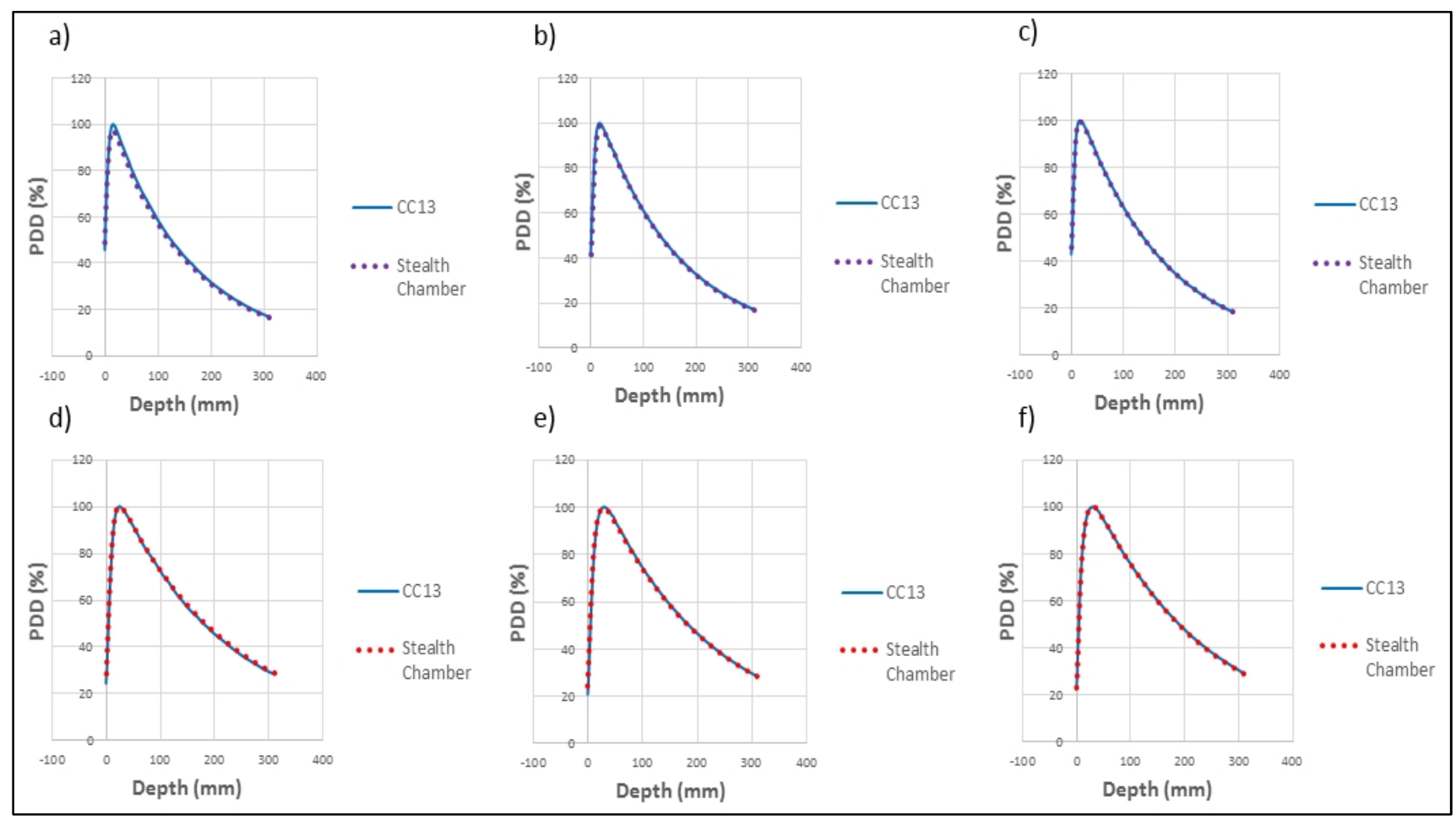

FIG. 6: Comparison of percent depth dose (PDD) for the stealth and regular ion chamber for $6 \mathrm{MV}$ a) $\left.1 \times 1 \mathrm{~cm}^{2} ; \mathrm{b}\right) 3 \times 3 \mathrm{~cm}^{2}$; and c) $5 \times 5 \mathrm{~cm}^{2}$ and 15 MV; d) $1 \times 1 \mathrm{~cm}^{2}$; e) $3 \times 3 \mathrm{~cm}^{2}$; and f) $5 \times 5 \mathrm{~cm}^{2}$. Agreement between PDD of both chambers holds across all field sizes. 

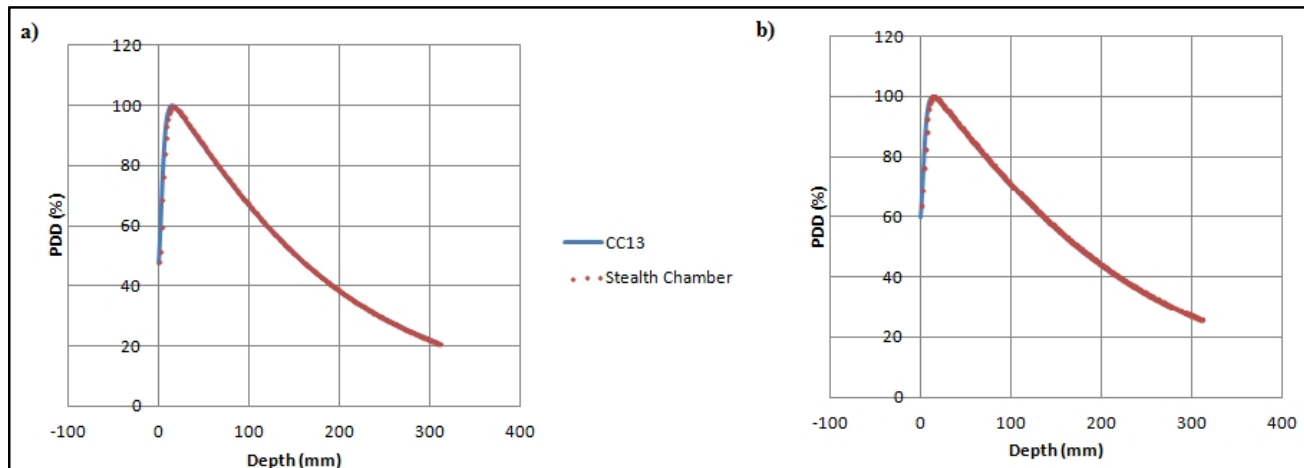

$-\mathrm{CC13}$

-.. Stealth Chamber

c)

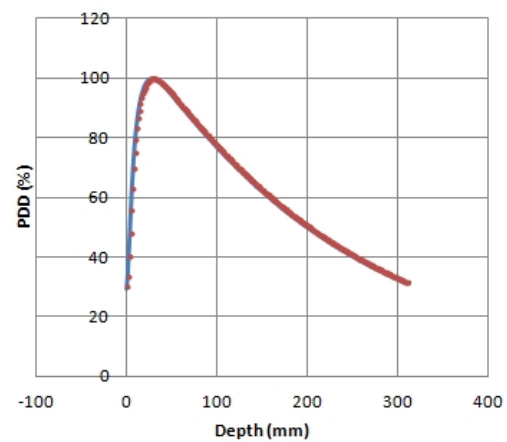

\section{$-\mathrm{cc} 13$}

-.. Stealth Chamber

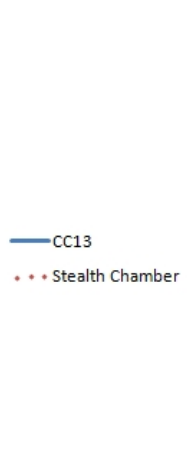

d)

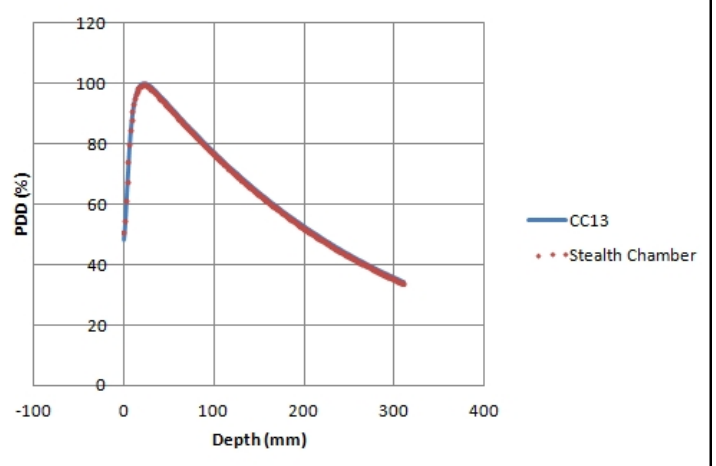

FIG. 7: Comparison of percent depth dose (PDD) for the stealth and regular ion chamber for $6 \mathrm{MV}$ a) $10 \times 10 \mathrm{~cm}^{2}$; b) $25 \times 25 \mathrm{~cm}^{2}$; and $15 \mathrm{MV}$; c) $10 \times 10 \mathrm{~cm}^{2}$; d) $25 \times 25 \mathrm{~cm}^{2}$.

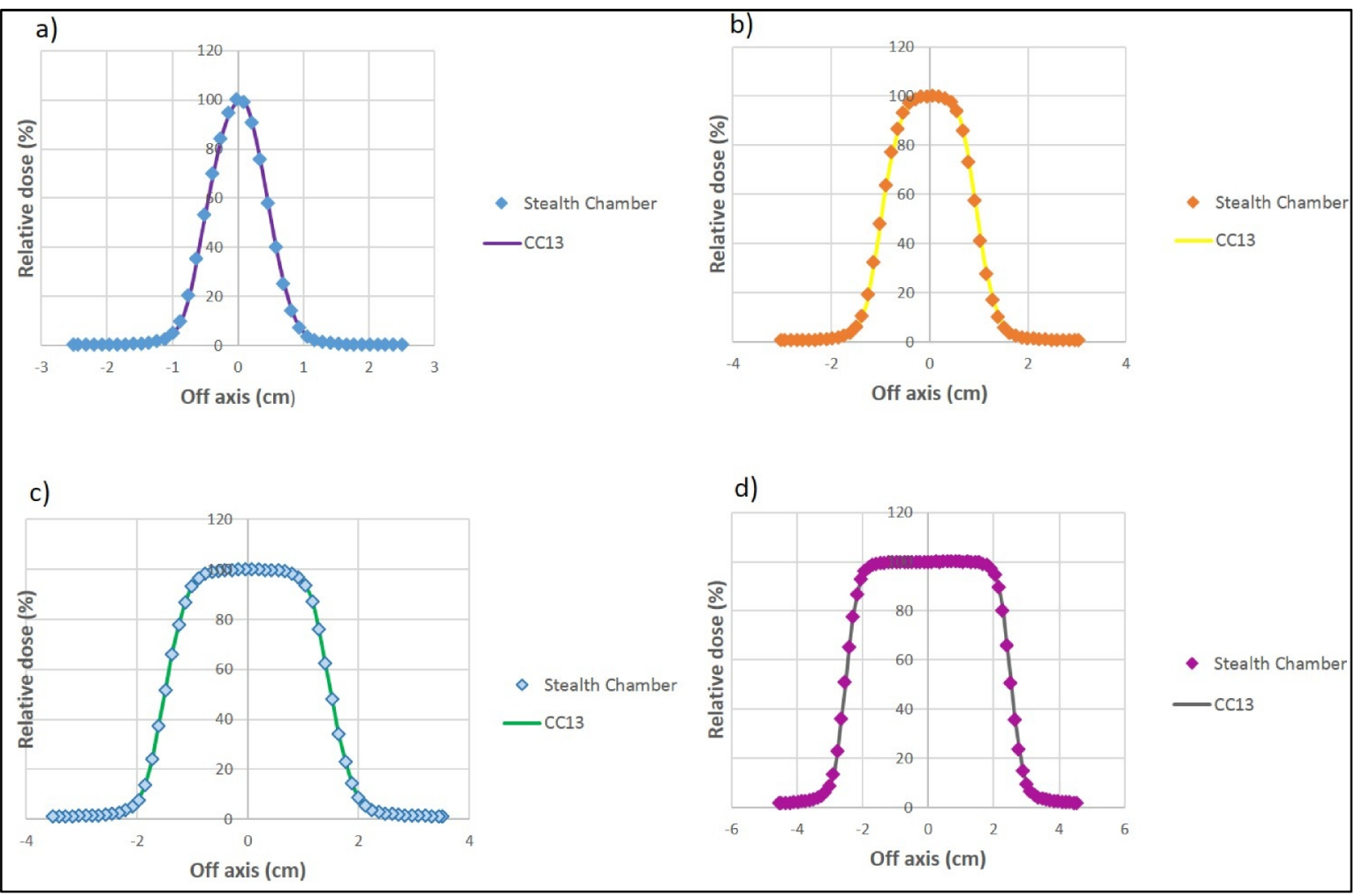

FIG. 8: Comparison of profiles of the stealth (diamond markers) and regular ion chamber (solid line) for $6 \mathrm{MV}$ at a depth of $1.5 \mathrm{~cm}$ across different field sizes a) $1 \times 1 \mathrm{~cm}^{2}$; b) $2 \times 2 \mathrm{~cm}^{2}$; c) $3 \times 3 \mathrm{~cm}^{2}$; and d) $5 \times 5 \mathrm{~cm}^{2}$. Agreement between measurements of both chambers holds across all field sizes. 
Agreement between PDD of both chambers holds across all field sizes. Figure 8 shows the results of the profile comparison for $1.5 \mathrm{~cm}$-depth measurements with $6 \mathrm{MV}$ and field sizes for $1 \times 1,2 \times 2,3 \times 3,5 \times 5 \mathrm{~cm}^{2}$. Figure 9 shows the results of the profile comparison for $10 \mathrm{~cm}$-depth measurements with 15 MV and field sizes for $10 \times 10,15 \times 15,20 \times 20$, and $25 \times 5 \mathrm{~cm}^{2}$. The same criterion of a $1 \%, 1 \mathrm{~mm}$ agreement was followed, and the data captured with the CC13 and the Stealth Chamber for the relative measurements of profiles are presented below. Similar results were observed with the rest of the fields and depths measured.
Figure 10 illustrates the transmission data obtained with the Matrixx (IBA) array with an open beam of $10 \times 10 \mathrm{~cm}^{2}$ for a 15 MV energy. Measurements with an open beam and with the Stealth Chamber mounted on the gantry of the linac were acquired. Measurements with a 6 and $15 \mathrm{MV}$ photon beam with field sizes ranging from $3 \times 3$ to $25 \times 25 \mathrm{~cm}^{2}$ were analyzed, and results similar to those shown in Figure 6 were obtained for profiles and gamma analysis with same passing criterion $(1 \%, 1 \mathrm{~mm})$. Average attenuations of $1.5 \%$ and $1.1 \%$ were measured by/on the central axis dose for energies corresponding to 6 and $15 \mathrm{MV}$, respectively. a)

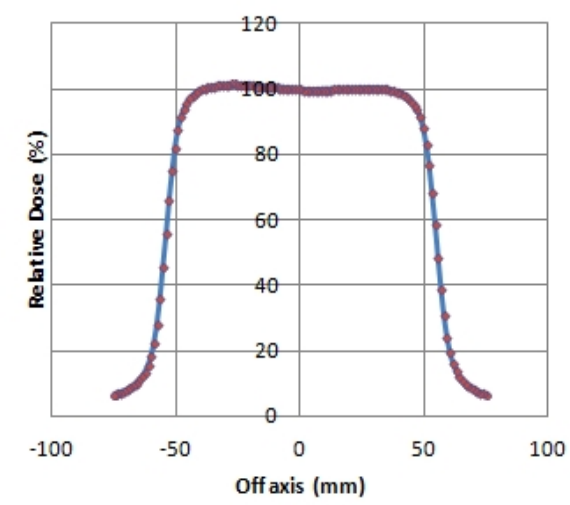

$\longrightarrow \mathrm{CC13}$

- Stealth Chamber

c)

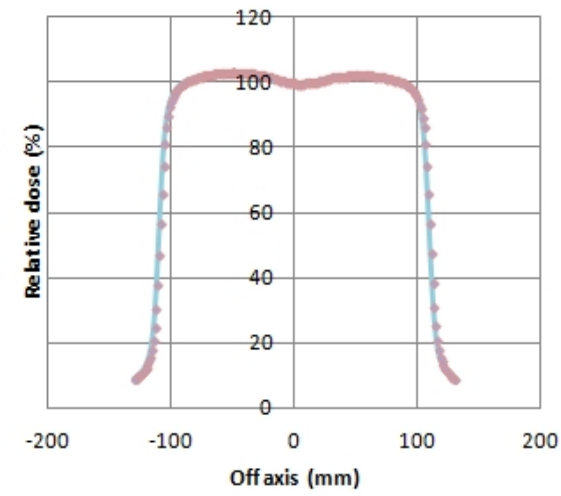

b)

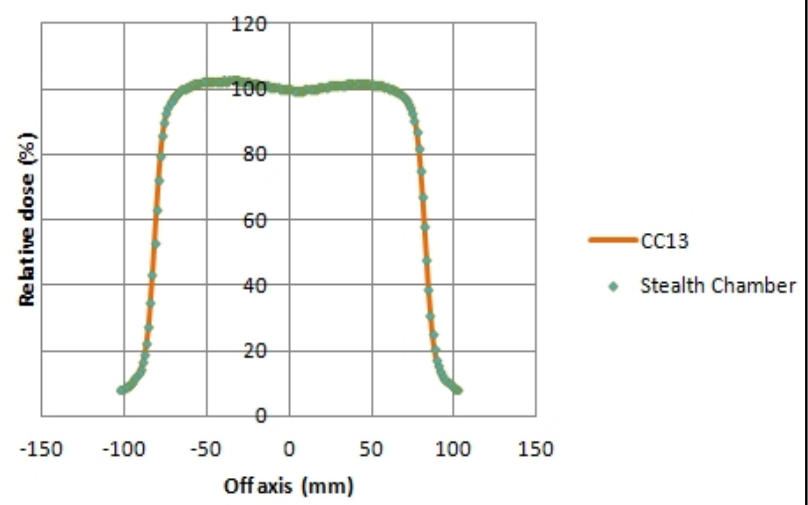

d)

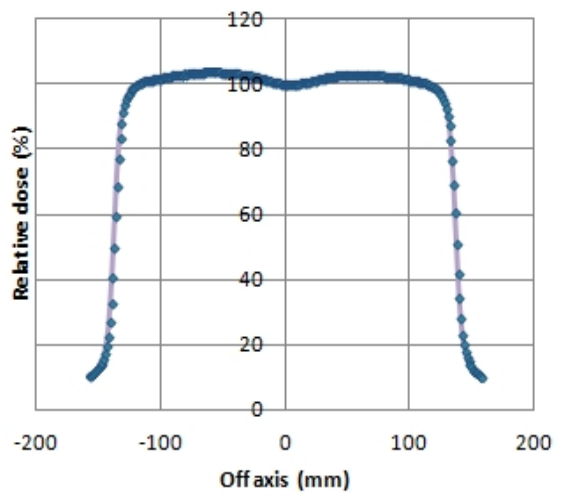

$-\mathrm{CC} 13$

- Stealth Chamber

FIG. 9: Comparison of profiles of the stealth (diamond markers) and regular ion chamber (solid line) for $15 \mathrm{MV}$ at a depth of $10 \mathrm{~cm}$ across different field sizes a) $10 \times 10 \mathrm{~cm}^{2}$; b) $15 \times 15 \mathrm{~cm}^{2}$; c) $20 \times 20 \mathrm{~cm}^{2}$ and d) $25 \times 25 \mathrm{~cm}^{2}$. Agreement between measurements of both chambers holds across all field sizes. 


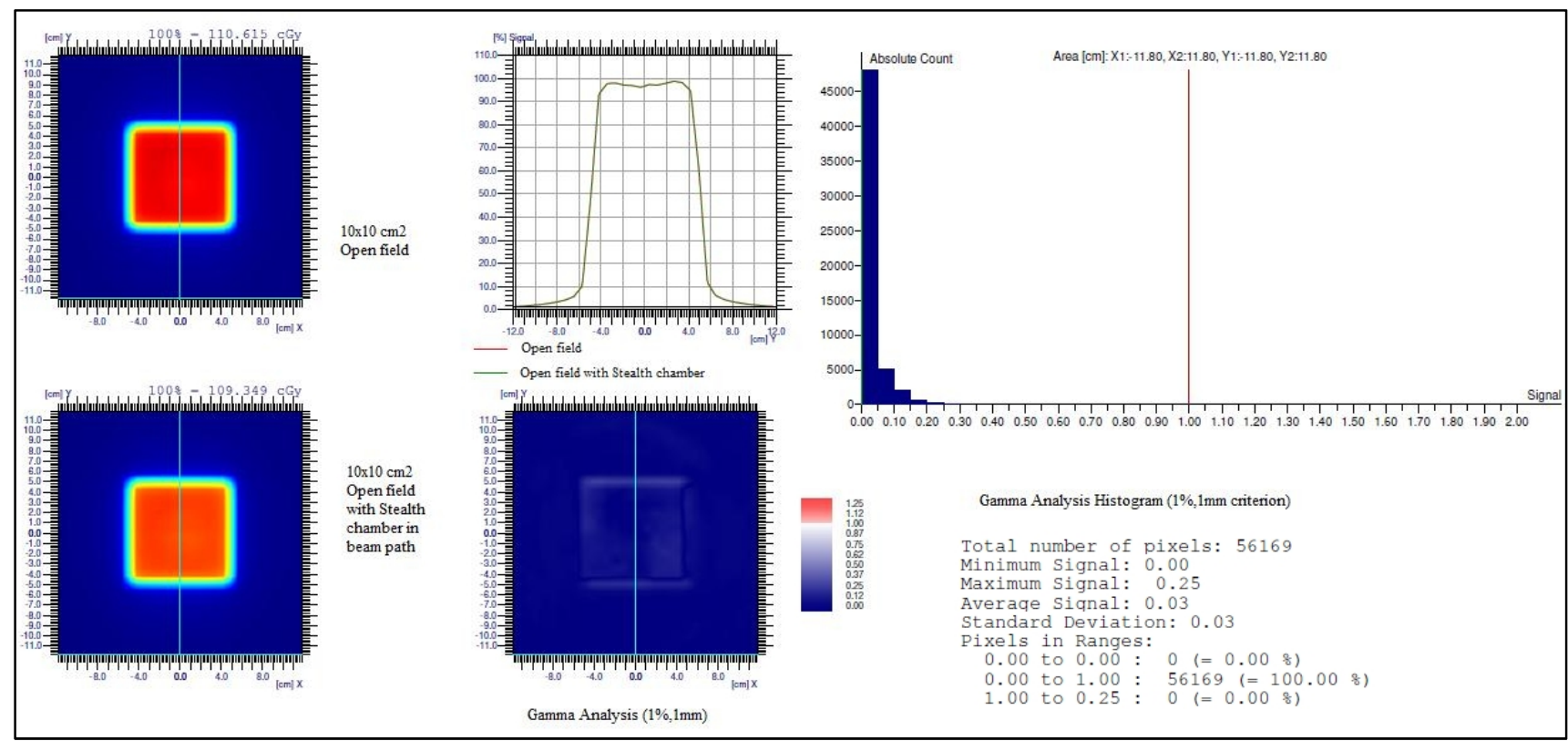

FIG. 10: Transmission data comparison with Matrixx (IBA) for a $10 \times 10 \mathrm{~cm}^{2}$ open field with and without the Stealth chamber in the beam path for a $15 \mathrm{MV}$ photon beam.

\section{Discussion}

Monte Carlo simulations of the Stealth Chamber in this investigation have shown that the data obtained with the Stealth Chamber as a reference chamber are comparable to those calculated with an open beam with no perturbation in the path of the beam. Furthermore, energy spectrum analyses of the phase-space files obtained from the simulations with and without the Stealth Chamber in the beam path have shown very little perturbation to the beam and for the majority of the spectrum can be considered null.

Experimental measurements of PDDs and profiles revealed comparable dosimetric data acquired with the Stealth Chamber and our gold standard, the CC13 chamber, as a reference and field chamber, respectively. When comparing the PDDs and profiles normalized to the dmax and central axis, respectively, the matching measurements of both systems reveal that the agreement holds for all measurements.

\section{Conclusion}

The theoretical and experimental results of this investigation indicate the benefits associated with accuracy and time expended during the acquisition of relative measurements as PDDs and profiles for beam commissioning when the Stealth Chamber is used as a reference chamber to perform these tasks. The perturbation in the presence of a small field due to the chamber is minimal and for practical purposes can be considered invisible to the photon beam. One of the Stealth Chamber's main advantages is the time saved due to its ability to attach to the head of the linac, thus eliminating the need to reposition it. The comparability of the Stealth Chamber re- sults and the gold standard (CC13) obtained in our investigations suggests that the Stealth Chamber has several advantages when used as a reference chamber for small-field dosimetry, and this makes it very suitable for use in the commissioning of a treatment planning system especially for stereotactic radiation therapy, where measurements of small-field data are critical for treatment planning system commissioning.

\section{Conflict of interest}

The authors declare that they have no conflicts of interest. The authors alone are responsible for the content and writing of the paper.

\section{Acknowledgements}

The authors would like to thank to Salih Arican, Eric Fleming and Juan Carlos Celi from IBA Dosimetry for their technical support during this investigation and Pooja Sarkar for her feedback.

\section{References}

1. Das I, Cheng C, Watts R, et al. Accelerator beam data commissioning equipment and procedures: Report of the TG-106 of the Therapy Physics Committee of the AAPM. Med Phys 2008: 35, 4186-215.

2. Wuerfel JU. Dose measurements in small fields. Medical Physics International 2013; 1: 81-90. 
3. Chetty I, Curran B, Cygler JE, et al. Issues associated with clinical implementation of Monte Carlo-based photon and electron external beam treatment planning. Med Phys 2007; 34:4818-52.

4. Vazquez-Quino LA, Massingill B, Shi C, et al. Monte Carlo modeling of a Novalis Tx Varian 6 MV with HD-120 multileaf collimator. J Appl Clin Med Phys 2012;13:3960.

5. Ahnesjo A. Collimator Scatter in Photon Therapy Beams. Med Phys 1995: 22: 267-78.

6. Ding G. Dose discrepancies between Monte Carlo calculations and measurements in the buildup region for a high-energy photon beam. Med Phys 2002; 29: 2459-63.

7. Ding GX, Duggan DM, Coffey CW. Commissioning stereotactic radiosurgery beams using both experimental and theoretical methods. Phys Med Biol 2006;51:2549-66.

8. Kijewski PK, Bjärngard BE, Petti PL. Monte Carlo calculations of scatter dose for small field sizes in a 60Co beam. Med Phys 1986;13:74-7.

9. Stathakis S, Esquivel C, Vazquez Quino L, et al. Accuracy of the Small Field Dosimetry Using the Acuros XB Dose Calculation Algorithm within and beyond Heterogeneous Media for 6 MV Photon Beams. International Journal of Medical Physics, Clinical Engineering and Radiation Oncology 2012;1:78-87.

10. Vazquez Quino L, Calvo O, Huerta C, et al. Monte Carlo simulations used to test the perturbation of a reference ion chamber prototype used for small fields. AAPM 2014 Annual Meeting (Poster), SU-E-T-242, 2014. http://www.aapm.org/meetings/2014am/PRAb s.asp? $\mathrm{mid}=90 \&$ aid $=23138$

11. Belosi MF, Rodriguez M, Fogliata A, et al. Monte Carlo simulation of TrueBeam flattening-filter-free beams using varian phase-space files: comparison with experimental data. $\mathrm{Med}$ Phys 2014;41:051707.

12. Gete E, Duzenli C, Milette MP, et al. A Monte Carlo approach to validation of FFF VMAT treatment plans for the TrueBeam linac. Med Phys 2013;40:021707.

13. Bergman AM, Gete E, Duzenli C, Teke T. Monte Carlo modeling of HD120 multileaf collimator on Varian TrueBeam linear accele- rator for verification of $6 \mathrm{X}$ and $6 \mathrm{X}$ FFF VMAT SABR treatment plans. J Appl Clin Med Phys 2014;15:4686.

14. Both JA, Pawlicki T. Monte Carlo Commissioning of Low Energy Electron Radiotherapy Beams using NXEGS Software. Int J Med Sci 2004; 1:63-75.

15. Craig J, Oliver M, Gladwish A, et al. Commissioning a fast Monte Carlo dose calculation algorithm for lung cancer treatment planning. $J$ Appl Clin Med Phys 2008;9:2702.

16. Gagné IM, Zavgorodni S. Evaluation of the analytical anisotropic algorithm in an extreme water-lung interface phantom using Monte Carlo dose calculations. J Appl Clin Med Phys 2006;8:33-46.

17. Kawrakow I, Walters BR. Efficient photon beam dose calculations using DOSXYZnrc with BEAMnrc. Med Phys 2006;33:3046-56.

18. Verhaegen F, Seuntjens J. Monte Carlo modelling of external radiotherapy photon beams. Phys Med Biol 2003; 48:R107-64.

19. Rogers DW, Faddegon BA, Ding GX, Ma CM, We J, Mackie TR. BEAM: a Monte Carlo code to simulate radiotherapy treatment units. $\mathrm{Med}$ Phys 1995;22:503-24.

20. Mora GM, Maio A, Rogers DW. Monte Carlo simulation of a typical 60Co therapy source. Med Phys 1999; 26:2494-502.

21. Kawrakow I. On the efficiency of photon beam treatment head simulations. Med Phys 2005;32:2320-26.

22. Kawrakow I. On the de-noising of Monte Carlo calculated dose distributions. Phys Med Biol 2002;47:3087-103.

23. Kry SF, Titt U, Pönisch F, Followill D, et al. A Monte Carlo model for calculating out-of-field dose from a varian $6 \mathrm{MV}$ beam. Med Phys 2006;33:4405-13.

24. Hasenbalg F, Fix MK, Born EJ, Mini R, Kawrakow I. VMC++ versus BEAMnrc: a comparison of simulated linear accelerator heads for photon beams. Med Phys 2008;35:1521-31.

25. Faddegon BA, Asai M, Perl J, et al. Benchmarking of Monte Carlo simulation of bremsstrahlung from thick targets at radiotherapy energies. Med Phys 2008;35:4308-17. 
26. Fragoso M, Kawrakow I, Faddegon BA, et al. Fast, accurate photon beam accelerator modeling using BEAMnrc: a systematic investigation of efficiency enhancing methods and cross-section data. Med Phys 2009;36:5451-66.

27. Borges C, Zarza-Moreno M, Heath E, et al. Monte Carlo modeling and simulations of the High Definition (HD-120) micro MLC and validation against measurements for a $6 \mathrm{MV}$ beam. Med Phys 2012;39:415-23.

28. Fix MK, Volken W, Frei D, et al. Monte Carlo implementation, and characterization of a 120 leaf MLC. Med Phys 2011;38:5311-20.

29. Gersh J. Stereotactic beam characterization using the IBA stealth chamber reference detector. IBA Dosimetry Whitepaper 2014. 